\title{
Case Report - Fetal Alcohol Exposure
}

\section{Carl C Bell}

Psychiatry and Public Health, University of Illinois, Chicago, USA

Corresponding author: Carl C Bell, Professor of Psychiatry and Public Health, University of Illinois, Chicago, USA; Tel: (773) 947-7310; E-mail: bell-carl@att.net

Received: Feb 17, 2016; Accepted: Mar 16, 2016; Published: Mar 18, 2016

Copyright: () 2016 Bell CC. This is an open-access article distributed under the terms of the Creative Commons Attribution License, which permits unrestricted use, distribution, and reproduction in any medium, provided the original author and source are credited.

\section{Abstract}

This case report highlights a clinical case of a child who was exposed to alcohol as a fetus, and, who as a result, developed learning and behavioral problems. For the past 45 years of practicing psychiatry the author has been seeing children who are usually referred from the school system for having problems learning and difficulty controlling their bad tempers. After years of attributing this behavior to various common psychological etiologies, e.g., having a male role model with a bad temper or being exposed to childhood violence, the author has come to understand while psychological etiologies remain important, the etiology of this constellation of symptoms may more likely due to an acquired biological cause. This case history highlights this new found understanding. Additionally, the intriguing etiology of the behavior seen in the presented case suggests that by knowing the etiologic cause of this common constellation of symptoms, it may be possible to use bio-technical strategies to ameliorate the problematic behaviors.

Keywords: Fetal alcohol; Psychological interventions; Nutraceuticals

\section{Introduction}

Since I was in medical school in 1968 [1], I have been seeing African-American children who have bad tempers, poor educational achievement, poor social skills, and poor judgment. In medical school these problems were referred to as mild mental retardation and Minimal Brain Dysfunction. A little more than ten years later, while working at the Chicago Board of Education, I saw 274 children who had these same problems to varying degrees, and by then these children were characterized by various labels, e.g., Trainably Mentally Handicapped (TMH), Educationally Mentally Handicapped (EMH), Minimal Brain Dysfunction, Neurosis, etc. because child psychiatric diagnosis was, like adult psychiatric diagnosis, becoming more descriptive in nature [2]. The trend for descriptive diagnoses was relevant at the time because it is rare in psychiatry to find a specific etiology for psychiatric illnesses and most problem behaviors were and still is caused by multiple factors. In psychiatry, we frequently do not know the etiology of the behaviors we observe that may constitute signs of mental illness or behavioral disturbances, yet we have to try to figure out medications and social or psychological interventions that ameliorate the mental illness or problem behaviors. However, as science progresses, as was done for cretinism and phenylketonuria, psychiatry's goal is to develop an etiologic understanding of as many psychiatric disorders as possible, because, it puts our profession in a better position to help the patients who come to us for treatment of their difficulties in life.

\section{Case History}

TJ (not the patient's real initials) is a 9-year-old AfricanAmerican male who was brought to the Jackson Park Hospital Family Medicine Center because of problems with his temper and learning in school. He had an Individualized Educational Plan (IEP) from the Chicago Board of Education which showed he was reading at a 2 nd grade level and his math was on the same level but a little worse (a 4th grade level would be average for a child his age). He was also reported to have extreme difficulty sitting still and paying attention in class. In addition, it was noted he had an explosive temper and was easily frustrated. The father reported his son had been previously diagnosed with Attention Deficit Hyperactivity Disorder (ADHD), and, although it helped a little, the stimulant medication (methylphenidate $10 \mathrm{mg}$ twice a day) had not helped his son a great deal. Nevertheless, the father was bringing his son into the clinic to get back on the medication as it did help a little.

On getting a birth history from the father, the father reports that the patient weighed 5 pounds and 5 ounces and he was a month and a half premature. He was also reported to have a heart murmur at birth. On questioning, the father also reports that the patient's mother was doing some social drinking (she did not know she was pregnant for two months) before she knew she was pregnant. He reports that he knew this because he was with her when she was drinking - they were drinking together and having sex. The patient had some vestiges of epicanthal folds, a flat mid-face, had an indistinct philtrum, and he seemed very immature for his age. Owing to the history of some improvement with the previous medication, the patient was given a prescription for the methylphenidate $10 \mathrm{mg}$ twice a day, but in addition the father was told that the patient had a Neurobehavioral Disorder associated with Prenatal Alcohol Exposure (ND-PAE) [3] not classical Attention 
Deficit Hyperactivity Disorder (ADHD). Furthermore, the father was also educated about emerging research about the animal studies and human studies using Nutraceuticals to improve the outcomes of subjects with ND-PAE. Lastly, the father was told that the Nutraceutical science was not yet rock solid, but, as their had been studies which showed that the use of these Nutraceuticals were safe, if it were the author's son I would put him on Choline 500 milligrams twice a day; Folate 400 micrograms twice a day, Vitamin A and $D(2,000$ International units and 200 International Units) Daily, and Omega - 3; 500 milligrams twice a day or 1,000 milligrams daily. The father and son were seen three months later and he reported that it had taken some time to find the Nutraceuticals, but, he had started the patient on the methylphenidate $10 \mathrm{mg}$ twice a day, and they were working as well as they had when he first put his son on medications. He also reported that the Chicago Public Schools system was continuing to work with his son to try to help with his problems with learning and poor emotional control. He was encouraged to get the Nutraceuticals or to enhance his son's source of Choline by getting him more egg yokes or beef liver. The patient and his father returned three months later. Since the local drug stores carried the Folate 400 micrograms, Vitamin A and D (2,000 International Units and 200 International Units), and Omega-3,500 milligrams, the father was able to purchase these Nutraceuticals and he had been able to order the Choline on line. He noted although the pills were rather large, his son had been able to learn to swallow them with little or no problem rather quickly. The father reported it seemed that his son was doing better and he based that improvement on the decrease in poor reports that he had been getting from his son's teacher. The patient reported that he did not really notice much difference. Three months later the father and son returned and the father reported that he was pretty sure that the Nutraceuticals were helping his son because he was able to reduce the patient's methylphenidate to just $10 \mathrm{mg}$ in the morning. The father confided he was not a big fan of placing his son on medication, and he liked the idea of Nutraceuticals and obtaining Choline in regular foods, but the only problem was that his son did not like egg yokes or beef liver and the Choline pills were the next best thing. I continue to follow the patient and he continues to improve although he is still taking the methylphenidate $10 \mathrm{mg}$ at $9 \mathrm{AM}$ that was prescribed for his symptoms of hyperactivity. His grades and math and reading scores have improved. If after a year of solid educational gains, I may discontinue the methylphenidate $10 \mathrm{mg}$ at $9 \mathrm{AM}$ and see how he does with just the Nutraceuticals.

\section{Discussion}

Currently, the rates of Neurobehavioral Disorder associated with Prenatal Alcohol Exposure (ND-PAE) in the United States in unknown. Partly this is due to the lack of epidemiological studies done the problem of Fetal Alcohol Spectrum Disorders (FASD) and the reality that the diagnosis of ND-PAE is brand new. The other problem is it is very embarrassing for the United States to have some populations to have ND-PAE at a much higher rate than others due to the social context highrisk populations live in in the United States. Work in the Cook
County Temporary Juvenile Detention Center and in the Chicago Board of Education's Special Education Services, where most of the children are African-American, suggests that $75 \%$ of the children in Juvenile Detention and Special Education have ND-PAE - the leading cause of intellectual disability [1]. The percentage of children who have ND-PAE and who wind up in child protective services is unknown but some estimates are that $15 \%$ to $28 \%$ [4] of these children were exposed to alcohol when they were in utero because their mother did not know they were pregnant and they were social drinking and having unprotected sex. However, other than a few studies on a few specific populations, the overall rates of ND-PAE in the US are unknown and the rates of this disorder in adults are unknown. Recently, May et al. published a paper on the prevalence of FASD in a predominately EuropeanAmerican, middle-class, Midwestern city and illustrated that the prevalence of FASD was much higher than expected - as high as nearly $5 \%$ in this population-based sample. However, it is likely that because of sociological contexts, there will be some populations that have much higher rates of ND-PAE than others. For example, our research revealed a rate on ND-PAE of $388 / 1,000$ in a low-income African-American community on Chicago's Southside of Chicago [4]. Since the only businesses that are thriving in low-income African-American communities all over the United States are liquor stores, it makes sense the rates of ND-PAE should be higher in such a community. It should not be a stretch to understand the concept of the "social determinants of health" [5]. Accordingly, it should be feasible to understand if there are two and three liquor/food stores every half mile, if about $50 \%$ of pregnancies are not planned [6], and if there is nothing wrong with social drinking, then women are going to mistakenly drink before they realize they are pregnant. To make the issue international is scope, there was a recent article published about the rate of Fetal Alcohol Syndrome in children living in remote Australia at 120/1,000 [7].

The results are disastrous. In the United States this has been going on for decades perhaps a century. We know the dominant culture in the United States used alcohol to control and destroy Native American culture and communities, but somehow we have missed that they did the same thing to African-Americans. Unfortunately, there is very little research done on African-Americans [8] and so we miss learning things that could improve everyone's well-being. We need to do some serious research on how many youth in child protective services, juvenile detention centers, and special education classrooms have the problem of ND-PAE as there may be something we can do to help them.

The good news is there is emerging scientific evidence that choline, an over the counter nutrient, may help to ameliorate the problem of ND-PAE. We know that alcohol denatures choline and animal studies show by giving animals who are pregnant alcohol causes their babies to have ND-PAE, but by supplementing the animal's diet with choline, and some other vitamins the problem on ND-PAE may be lessened $[9,10]$. There are also studies on humans that indicate both prenatal and postnatal choline may lessen the difficulties that prenatal alcohol exposure does in humans [11-14]. 
Accordingly, there is evolving research that suggests Nutraceuticals may be helpful in humans prenatally and after the child is born. We are still waiting for absolute sure proof of this hypothesis, but it makes sense based on an understanding of embryology, biochemistry, and epigenetics. It would certainly be better to give patients with ND-PAE who have a plethora of learning, judgment, and emotional problems, and who have a poor response to psychiatric medications, therapeutic vitamins (Nutraceuticals) to help their damaged brains get back on a normal developmental track. The practice of medicine is clear that the human body is always trying to heal itself; we just need not to cause harm and to supply the body with what it needs to heal. The research on choline and other vitamins is going to take a long time to prove, but, in the meantime as this case history presents, clinicians can explore the use of Nutraceuticals with patients who have ND-PAE to see if it in fact benefits their outcomes.

\section{References}

1. Bell CC (2014) Fetal alcohol exposure among African Americans. Psychiatr Serv 65: 569.

2. American Psychiatric Association (1980) The Diagnostic and Statistical Manual (3rdedn). Washington DC: American Psychiatric Press, Inc.

3. American Psychiatric Association (2013) The Diagnostic and Statistical Manual (5thedn). Washington DC: American Psychiatric Press, Inc.

4. Bell CC, Chimata R (2015) "Prevalence of Neurodevelopmental Disorders in Low-Income African-Americans at a Family Medicine Clinic on Chicago's Southside." Psychiatric Services 66: 539-542.

5. Marmot, Michael and Richard G. Wilkinson (2005) Social Determinants of Health (2ndedn). Oxford: Oxford Universities Press.
6. Finer LB, Zolna MR (2011) Unintended pregnancy in the United States: incidence and disparities, Contraception 84: 478-485.

7. Fitzpatrick JP, Jane L, Maureen C, June O, Manuela LF, et al. (2015) Prevalence of fetal alcohol syndrome in a populationbased sample of children living in remote Australia: The Lililwan* Project. Journal of Paediatrics and Child Health 51: 450-457.

8. US Department of Health and Human Services (2001) Mental Health: Culture, Race, and Ethnicity: A Supplement to Mental Health: A Report of the Surgeon General. Rockville, MD: US Department of Health and Human Services, US Public Health Service.

9. Thomas JD, Jeremy SB, Bryan KAO, Neill TMO (2007) Choline supplementation following third-trimester-equivalent alcohol exposure attenuates behavioral alterations in rats. Behavioral Neuroscience 121: 120-130.

10. Jennifer T, Abou EJ, Hector DD (2009) Prenatal choline supplementation mitigates the adverse effects of prenatal alcohol exposure on development in rats. Neurotoxicology and Teratology 31: 303-311.

11. Ross Randal G, Hunter SK, Hoffman C, Mc Carthy L (2015) Perinatal phosphatidylcholine supplementation and early childhood behavior problems: Evidence for CHRNA7 moderation. American Journal of Psychiatry.

12. Ross RG, Sharon KH, Beuler LJ (2013) Perinatal choline effects on neonatal Pathophysiology related to later schizophrenia risk. American Journal of Psychiatry 170: 290-298.

13. Wozniak JR, Anita JF, Judith KE, Birgit AF (2015) Choline supplementation in children with fetal alcohol spectrum disorders: a randomized, double-blind, placebo-controlled trial. American Journal of Clinical Nutrition 102: 1113-1125.

14. Wozniak JR, Anita JF, Judith KE, Maria GK (2013) Choline supplementation in children with fetal alcohol spectrum disorders has high feasibility and tolerability. Nutrition Research 33: 897-904. 